\title{
Blocking of Proteolytic Processing and Deletion of Glycosaminoglycan Side Chain of Mouse DMP1 by Substituting Critical Amino Acid Residues
}

\author{
Tao Peng ${ }^{a}$ Bingzhen Huang ${ }^{a}$ Yao Sun ${ }^{a}$ Yongbo Lu ${ }^{b}$ Lynda Bonewald ${ }^{b}$ \\ Shuo Chen ${ }^{c}$ William T. Butler ${ }^{a}$ Jerry Q. Feng ${ }^{a}$ Rena N. D'Souza ${ }^{a}$ Chunlin Qin $^{a}$ \\ a Department of Biomedical Science, Baylor College of Dentistry, Texas A\&M University System Health Science \\ Center, Dallas, Tex., 'b School of Dentistry, University of Missouri at Kansas City, Kansas City, Mo., and ' School of \\ Dentistry, University of Texas Health Science Center, San Antonio, Tex., USA
}

\section{Key Words}

Dentin matrix protein $1 \cdot$ Proteolytic processing $\cdot$

Glycosaminoglycan · Mutation

\begin{abstract}
Dentin matrix protein 1 (DMP1) is present in the extracellular matrix (ECM) of dentin and bone as processed $\mathrm{NH}_{2}$ and $\mathrm{COOH}$-terminal fragments, resulting from proteolytic cleavage at the $\mathrm{NH}_{2}$ termini of 4 aspartic acid residues during rat DMP1 processing. One cleavage site residue, Asp ${ }^{181}$ (corresponding to $\mathrm{Asp}^{197}$ of mouse DMP1), and its flanking region are highly conserved across species. We speculate that cleavage at the $\mathrm{NH}_{2}$ terminus of $\mathrm{Asp}^{197}$ of mouse DMP1 represents an initial, first-step scission in the whole cascade of proteolytic processing. To test if $\mathrm{Asp}^{197}$ is critical for initiating the proteolytic processing of mouse DMP1, we substituted Asp ${ }^{197}$ with Ala ${ }^{197}$ by mutating the corresponding nucleotides of mouse cDNA that encode this amino acid residue. This mutant DMP1 cDNA was cloned into a pcDNA3.1 vector. Data from transfection experiments indicated that this single substitution blocked the proteolytic processing of mouse DMP1 in HEK-293 cells, indicating that cleavage at the $\mathrm{NH}_{2}$ terminus of $\mathrm{Asp}^{197}$ is essential for exposing other cleavage sites for the conversion of DMP1 to its fragments. The $\mathrm{NH}_{2}-$ terminal fragment of DMP1 occurs as a proteoglycan form (DMP1-PG) that contains a glycosaminoglycan (GAG) chain.
\end{abstract}

Previously, we showed that a GAG chain is linked to $\operatorname{Ser}^{74}$ in rat DMP1 (Ser ${ }^{89}$ in mouse DMP1). To confirm that mouse DMP1-PG possesses a single GAG chain attached to $\mathrm{Ser}^{89}$, we substituted $\mathrm{Ser}^{89}$ by Gly ${ }^{89}$. Data from transfection analysis indicated that this substitution completely prevented formation of the GAG-containing form, confirming that DMP1PG contains a single GAG chain attached to $\mathrm{Ser}^{89}$ in mouse DMP1.

Copyright $\odot 2008$ S. Karger AG, Basel

\section{Introduction}

Dentin matrix protein 1 (DMP1) was discovered by cDNA cloning [George et al., 1993]. The distinctive feature of DMP1 is a large number of acidic domains, a property that implicates it is a possible participant in regulat-

\begin{tabular}{ll}
\hline Abbreviations used in this paper \\
\hline DMP1 & dentin matrix protein 1 \\
DMP1-PG & proteoglycan form of DMP1 \\
ECM & extracellular matrix \\
GAG & glycosaminoglycan \\
HEK-293 & human epithelial kidney 293 cell line
\end{tabular}

Dr. Chunlin Qin

Department of Biomedical Sciences, Baylor College of Dentistry

Texas A\&M University System Health Science Center

3302 Gaston Avenue, Dallas, TX 75246 (USA)

Tel. +1 214828 8292, Fax +1 214874 4538, E-Mail cqin@bcd.tamhsc.edu 
ing matrix mineralization. This purported biological function is supported by the observations that MC3T3E1 cells overexpressing DMP1 demonstrate an earlier onset of mineralization and a significantly larger size of the induced mineralized nodules compared to those of the control cells [Narayanan et al., 2001]. Findings from Dmp1-null mice strengthen the conclusion that DMP1 plays an important role in biomineralization [Ye et al., $2004,2005]$. Recent studies also demonstrated the association between mutations in the DMP1 gene and autosomal recessive hypophosphatemic rickets, a novel human disorder [Feng et al., 2006; Lorenz-Depiereux et al., 2006].

DMP1 was originally postulated to be dentin specific, but later its expression was observed in bone and nonmineralized tissues [D'Souza et al., 1997; MacDougall et al., 1998; Ogbureke et al., 2007]. The cDNA of DMP1 has been cloned and sequenced in a number of species including rat [George et al., 1993], mouse [MacDougall et al., 1998], human [Hirst et al., 1997] and crocodilia [Toyosawa et al., 1999]. After translation, rat DMP1 is proteolytically processed into an $\mathrm{NH}_{2}$-terminal $(37-\mathrm{kDa})$ fragment and a $\mathrm{COOH}$-terminal $(57-\mathrm{kDa})$ fragment at 4 bonds, $\mathrm{Phe}^{173}$-Asp ${ }^{174}$, Ser ${ }^{180}-\mathrm{Asp}^{181}$ Ser $^{217}-\mathrm{Asp}^{218}$ and $\mathrm{Gln}^{221}-\mathrm{Asp}^{222}$, in the amino acid sequence [Qin et al., 2003]. Amino acid sequence alignment of DMP1 shows that residues in the region flanking $\mathrm{Ser}^{180}$-Asp ${ }^{181}$ are highly conserved across a very broad range of species, indicating that the proteolytic cleavage at this site must be related to an important biological function [Qin et al., 2004].

More recently, our group discovered that, in addition to the $37-\mathrm{kDa}$ form, the $\mathrm{NH}_{2}$-terminal fragment of DMP1 also occurs as a proteoglycan, present in the extracellular matrix (ECM) of bone and dentin [Qin et al., 2006]. The proteoglycan variant, referred to as DMP1-PG, possesses a glycosaminoglycan (GAG) chain that is made predominantly of chondroitin-4-sulfate and is linked to the core protein via $\mathrm{Ser}^{74}$ in rat DMP1.

The purpose of this investigation was to determine the functional role of $\mathrm{Asp}^{197}$ of mouse DMP1 (corresponding to $A s p^{181}$ in rat DMP1) as a key cleavage site residue essential for initiating the proteolytic processing events of DMP1. We also analyzed whether $\operatorname{Ser}^{89}$ of mouse DMP1 $\left(\mathrm{Ser}^{74}\right.$ in rat DMP1) is the only GAG chain attachment site. In our study, the cleavage site residue $\mathrm{Asp}^{197}$ of mouse DMP1 was substituted by Ala ${ }^{197}$ (D197A substitution), while the $\operatorname{Ser}^{89}$ residue was substituted by Gly ${ }^{89}$ (S89G substitution). Results from transfection experiments demonstrated that D197A substitution prevented the pro- teolytic processing, while S89G substitution completely prevented formation of the glycosaminoglycan form of mouse DMP1.

\section{Materials and Methods}

\section{Plasmid Construction}

For generation of a DMP1 cDNA construct, we utilized a pBC$\mathrm{KS}+$ construct carrying full-length mouse DMP1 cDNA and a pcDNA3.1 vector (Invitrogen, San Diego, Calif., USA). Mutagenesis was performed on the $\mathrm{pBC}-\mathrm{KS}+$ construct to create 3 types of mutant DMP1 cDNA, using the QuikChange II Site-Directed Mutagenesis Kit (Stratagene, La Jolla, Calif., USA) following the manufacturer's protocol. For substitution of residues at cleavage sites, $\mathrm{Asp}^{197}$ and $\mathrm{Asp}^{234}$ in mouse DMP1 were substituted by Ala ${ }^{197}$ and $\mathrm{Ala}^{234}$, respectively; these 2 substitutions were named D197A and D234A, respectively. For the substitution of GAG chain attachment residue, Ser ${ }^{89}$ was substituted by Gly ${ }^{89}$ (designated S89G substitution). These mutations were confirmed by restriction enzyme digestion and DNA sequencing. The mutant DMP1 cDNA fragments were released by EcoRI digestion, and subcloned into pcDNA3.1 vectors.

\section{Cell Culture, Transient Transfection and Western} Immunoblotting

The human epithelial kidney 293 (HEK-293) cells were selected for transient transfection, since these cells have a high transfection efficiency for mammalian expression vector, and in our preliminary experiments, DMP1 was clearly detected when these cells were used for transfection. The HEK-293 cells were grown in Dulbecco's modified Eagle's medium (GIBCO-BRL/Invitrogen, Carlsbad, Calif., USA) supplemented with $10 \%$ (v/v) fetal bovine serum, 2 mM L-glutamine. The pcDNA3.1 constructs containing DNA fragments coding for normal DMP1 as well as those for D197A, D234A and S89G substitutions were transfected into HEK-293 cells using the Lipofectamine 2000 (Invitrogen, Carlsbad, Calif., USA) method. After 16-18 h of transfection, cells were changed to conditioned medium, which was harvested at $72 \mathrm{~h}$. Lyophilized conditioned media from HEK-293 cells or samples containing DMP1 and its processed fragments purified by ion exchange chromatography were separated on 4-20\% Tris-glycine SDS-PAGE gels (LifeGels). For isolation of DMP1 and its fragments, the culture medium was loaded onto a Q-Sepharose column (Amersham Biosciences, Piscataway, N.J., USA) connected to a fast protein liquid chromatography system. In this separation, DMP1 and its processed fragments co-elute in early fractions, while DMP1-PG elutes in late fractions.

Proteins separated on SDS-PAGE were visualized by StainsAll staining and/or Western immunoblotting. The following 3 types of antibodies were used for chemiluminescent Western immunoblotting. (1) A polyclonal antibody generated by using peptide AYNHKPIGDQDDNDC (residue 471-485 of mouse DMP1) was used to detect the carboxyl terminus of DMP1; this antibody (designated anti-DMP1-C-785) recognizes the 57-kDa fragment as well as the full-length form of DMP1. (2) A polyclonal antibody raised against peptide GLGPEEGQWGGPSKLDSDEDS (residue 101-121 of mouse DMP1) recognizes the amino terminus of DMP1; this antibody (referred to as anti-DMP1-N-784) is immu- 

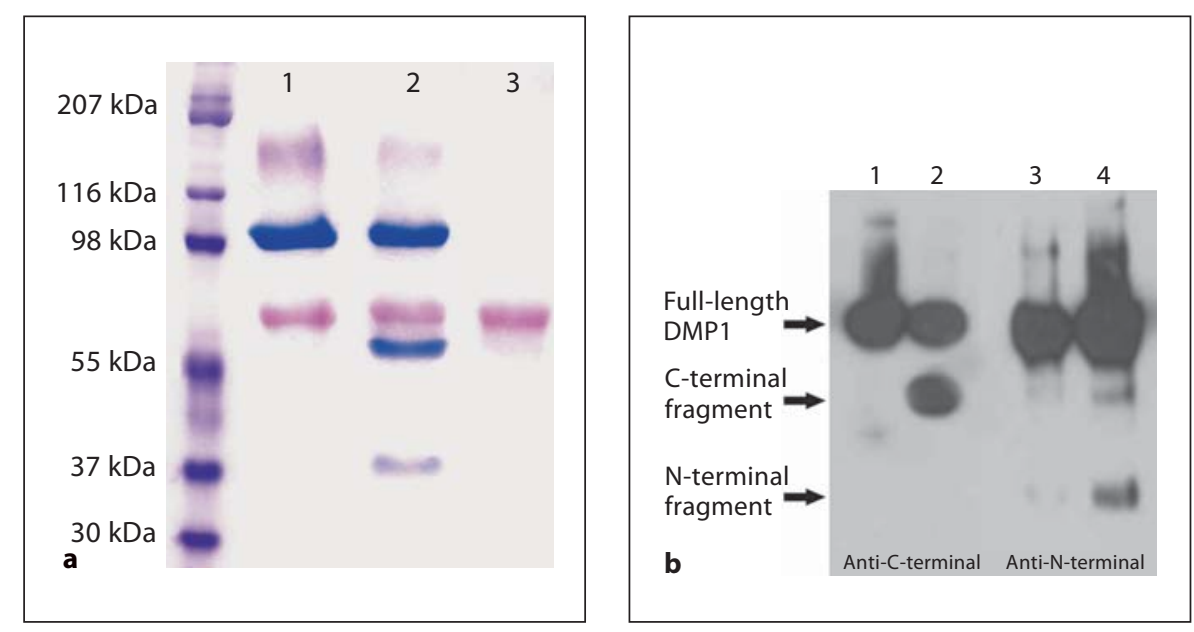

Fig. 1. Substitution of a single amino acid residue prevented cleavage of mouse DMP1. a Stains-All staining. Lane 1: conditioned culture medium of cells transfected with pcDNA3.1 construct carrying a mouse DMP1 cDNA that encodes DMP1 protein, in which $\mathrm{Asp}^{197}$, an amino acid residue at a key cleavage site, was substituted by Ala ${ }^{197}$ (note the absence of the lower molecular weight, blue-stained band). Lane 2: pcDNA3.1 vector carrying normal mouse DMP1 cDNA (note the presence of low molecular weight bands stained blue). Lane 3: empty pcDNA3.1 vector (without DMP1 cDNA). The blue band at approximately $100-110 \mathrm{kDa}$ is full-length DMP1. Blue bands at approximately 57 and $37 \mathrm{kDa}$ are DMP1 fragments. Note that the $37-\mathrm{kD}$ a fragment did not stain as darkly as the $57-\mathrm{kDa}$ fragment, which is most likely due to the less acidic properties of the $37-\mathrm{kDa}$ fragment. The purple band at approximately $65 \mathrm{kDa}$ represents BSA from the culture medium. For each lane, $600 \mu \mathrm{l}$ of lyophilized cell-conditional culture medium was loaded onto SDS-PAGE gels. b Western immunoblotting using polyclonal antibodies against $\mathrm{COOH}$-terminal region of DMP1 (left 2 lanes, anti-DMP1-C-785) and $\mathrm{NH}_{2}$-terminal region of DMP1 (right 2 lanes, anti-DMP1-N-784). Lanes 1 and 3 are the same sample (D197A) as in lane 1 of a; lanes 2 and 4 are the same as in lane 2 of a (DMP1 without mutation). Note the absence of the DMP1 fragments in the lanes with an amino acid substitution at the proposed cleavage site (lanes 1 and 3). For each lane, $300 \mu \mathrm{l}$ of lyophilized cell-conditioned culture medium was loaded onto SDS-PAGE gels. Please note that DMP1-PG was hardly recognized by the polyclonal antibody against the $\mathrm{NH}_{2}$-terminal region of DMP1.
Fig. 2. D234A substitution did not block DMP1 processing. For these experiments, proteins (DMP1 and its processed fragments) purified by ion exchange chromatography were used. Note that DMP1-PG was eluted in different chromatographic fractions and thus was not included in these samples. a About $6 \mu \mathrm{g}$ of purified protein was loaded onto each lane for Stains-All staining. Note that the pattern of protein bands is similar between the D234A substitution (lane 1) and the normal control group (lane 2). b About $3 \mu \mathrm{g}$ of purified protein was loaded onto each lane for Western immunoblotting using the antibody against the $\mathrm{COOH}$-terminal region of mouse DMP1 (anti-DMP1-C785). Lane 1: D234A substitution; lane 2: normal control group. Note the presence of the DMP1 COOH-terminal fragment (approximately $57 \mathrm{kDa}$ ) in both groups.
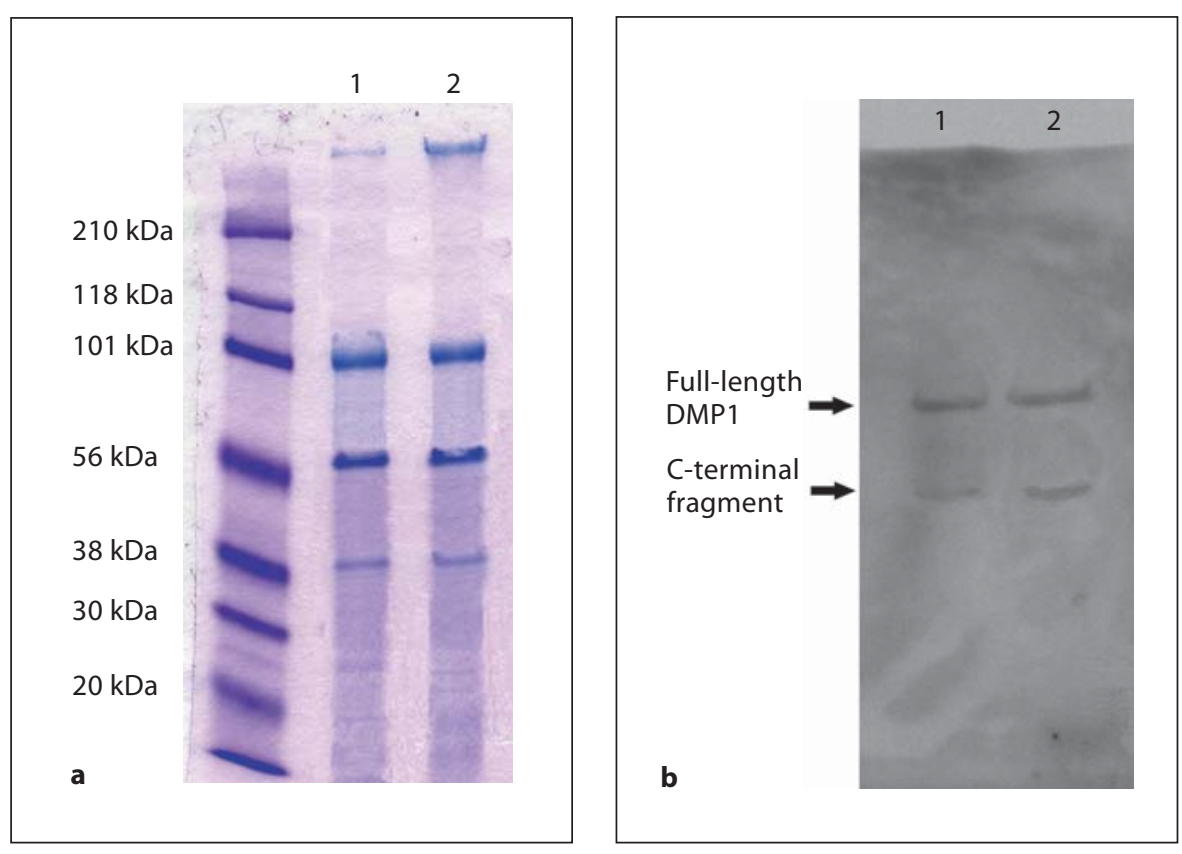


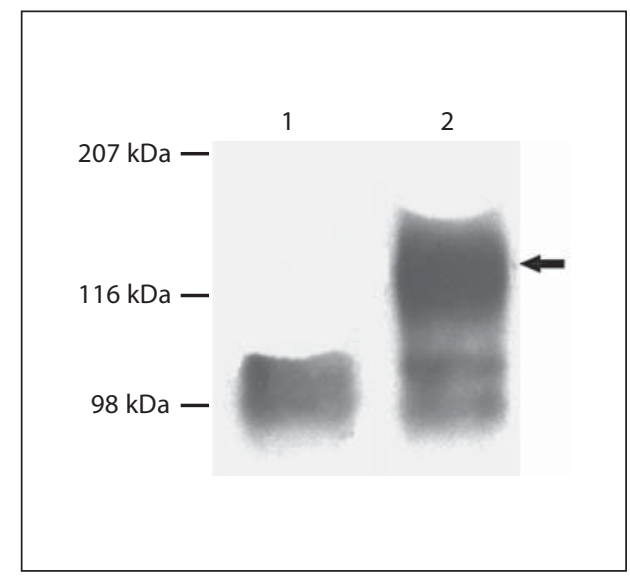

Fig. 3. Substitution of $\mathrm{Ser}^{89}$ in mouse DMP1 completely prevented GAG glycosylation of DMP1. $300 \mu \mathrm{l}$ of cell-conditioned culture medium was loaded onto SDS-PAGE, and the proteins were visualized by Western immunoblotting using a monoclonal antibody against the $\mathrm{NH}_{2}$-terminal region of DMP1 (anti-DMP1-N-clone 9B6.3). This monoclonal antibody [Qin et al., 2006] shows a strong reaction to DMP1-PG, but a weak reaction to the $37-\mathrm{kDa}$ form. Lane 1: conditioned culture medium from cells transfected with the pcDNA3.1 construct carrying a mutant cDNA that encodes mouse DMP1, in which the GAG attachment residue, $\mathrm{Ser}^{89}$, was substituted by Gly ${ }^{89}$ (S89G). Lane 2: conditioned culture medium from cells transfected with the pcDNA3.1 construct carrying normal mouse DMP1 cDNA. Note the absence of DMP1-PG in lane 1 compared to the intact proteoglycan in lane 2 (arrow).

noreactive to the $37-\mathrm{kDa}$ fragment and the full-length form of DMP1, but shows a relatively weak reaction to DMP1-PG. (3) A monoclonal antibody (named anti-DMP1-N-clone 9B6.3) generated using the $\mathrm{NH}_{2}$-terminal fragment of DMP1 isolated from rat long bone as the antigen is strongly reactive to DMP1-PG, but shows a relatively weak reaction to the $37-\mathrm{kDa}$ form; previously, this monoclonal antibody was used to successfully isolate DMP1PG from bone [Qin et al., 2006]. All 3 antibodies were purified by affinity columns.

\section{Results}

D197A Substitution Blocked the Proteolytic Processing of Mouse DMP1

Stains-All staining as well as Western immunoblotting showed that the conditioned culture media of HEK293 cells transfected with the pcDNA3.1 construct carrying either normal mouse DMP1 cDNA or mutant DMP1 contained significant amounts of DMP1 and/or its fragments, whereas the media from cells transfected with empty vector or with vector carrying the DMP1 cDNA insert in the incorrect orientation did not contain any DMP1 or its fragments.

For analysis of the product of the D197A substitution, conditioned culture medium from cells transfected with a construct carrying the D197A substitution as well as that from the normal control were directly loaded onto SDS-PAGE gels for Stains-All staining and Western immunoblotting. As shown in figure 1a, an approximately $100-\mathrm{kDa}$ band (stained blue) representing full-length DMP1 as well as the $57-$ and $37-\mathrm{kDa}$ fragments were clearly visible in the normal control, whereas in the D197A substitution group, only full-length DMP1 was observed. The findings indicate that the D197A substitution blocked the conversion of DMP1 into N- and C-terminal fragments. The result from Western immunoblotting (fig. 1b) using the anti-DMP1-C-785 and anti-DMP1-N-784 polyclonal antibodies further confirmed the above findings. It should be noted that in the normal control group, a significant amount of uncleaved, full-length DMP1 was observed. This is most likely due to overexpression of the protein driven by the cytomegalovirus promoter in excess amounts that could not be cleaved by endogenous protease.

\section{D234A Substitution Did Not Block the DMP1}

\section{Processing}

For analysis of the D234A substitution, proteins from the conditioned culture medium of cells transfected with a construct carrying the D234A substitution as well as from the normal control was first purified by ion exchange chromatography, and then fractions containing DMP1 and its fragments, were loaded onto SDS-PAGE gels for Stains-All staining and Western immunoblotting. Both Stains-All staining (fig. 2a) and Western immunoblotting (fig. $2 \mathrm{~b}$ ) of the D234A substitution and normal control groups showed essentially identical protein bands, indicating that the D234A substitution had no effect on the proteolytic processing of DMP1.

\section{S89G Substitution Prevented GAG Glycosylation of $D M P 1$}

For analysis of the S89G substitution, conditioned culture medium from cells transfected with a construct carrying the S89G substitution as well as from the normal control was directly loaded onto SDS-PAGE gels for Western immunoblotting. As shown in figure 3, Western immunoblotting using the anti-DMP1-N-clone 9B6.3 antibody demonstrated the presence of DMP1-PG, which appeared as broad bands in the normal control, whereas these high molecular weight bands were completely ab- 
sent in the S89G substitution group. This observation confirmed that $\mathrm{Ser}^{89}$ is the only site linking a single GAG chain to the core protein.

\section{Discussion}

In the ECM of bone and dentin, 3 forms of DMP1 have been identified: (1) the $\mathrm{NH}_{2}$-terminal fragment, (2) the $\mathrm{COOH}$-terminal fragment and (3) DMP1-PG. Based on a number of observations, we have proposed that proteolytic processing of DMP1 is one of the necessary steps in the biomineralization of normal bones and teeth [Qin et al., 2004]. We believe that these variants with distinct biochemical features may play different roles in biomineralization. In vitro mineralization studies have demonstrated that the $\mathrm{COOH}$-terminal fragment promotes mineralization by acting as a nucleator for hydroxyapatite formation [Tartaix et al., 2004, He et al., 2005; Gajjeraman et al., 2007]. Information regarding the function of DMP1-PG is lacking.

Our previous studies showed that all 4 cleavage sites in DMP1 occur at the $\mathrm{NH}_{2}$ termini of aspartyl residues. In this investigation we tested whether Asp ${ }^{197}$ of mouse DMP1 is a key cleavage site residue, essential for initiating proteolytic processing of DMP1. Our findings showed that substitution of Asp ${ }^{197}$ by Ala ${ }^{197}$ blocked the proteolytic cleavage of DMP1, whereas the substitution of another cleavage site residue, Asp ${ }^{234}$, did not prevent proteolytic processing of DMP1. These findings further strengthened our theory that DMP1 processing is an activation step. Based on these observations, we envision that cleavage at the $\mathrm{NH}_{2}$ terminus of $\mathrm{Asp}^{197}$ represents an initial, first-step scission in the whole cascade of DMP1 processing. It is likely that conformational changes after the initial cleavage at $\mathrm{Asp}^{197}$ expose other sites for the proteinase(s) to exert its scission; that is, breaking of the bond Ser ${ }^{196}$-Asp ${ }^{197}$ starts a chain of proteolytic events, resulting in cleavage of several other peptide bonds. Thus, the cleavage of the Ser ${ }^{196}$-Asp ${ }^{197}$ bond would be essential for the release of DMP1 fragments from the precursor, and this step would be critical for the biological functions of DMP1.

We did not detect DMP1 in the culture medium of nontransfected HEK-293 cells. The observation that normal DMP1 is processed in these cells indicated that the proteinase(s) responsible for cleaving DMP1 must exist in a very broad range of tissues or cells. However, the cleavage pattern of DMP1 in HEK-293 cells showed certain differences from that of DMP1 in bone or dentin ECM; in the latter 2 tissue compartments, DMP1 fragments appeared as clusters of bands indicating multiple cleavages, while the protein band pattern in the medium of HEK293 cells suggested a single cleavage. It is possible that in the ECM of bone and dentin, more than 1 proteinase is involved in DMP1 processing. Further studies are warranted to identify the enzymes responsible for DMP1 processing.

A careful analysis of the amino acid sequence of mouse DMP1 shows that $\mathrm{Ser}^{89}$-Gly ${ }^{90}$ is 1 of only 2 Ser-Gly dipeptides, an amino acid motif specific for the attachment of GAG chains to serine. The finding that the S89G substitution completely eliminated the proteoglycan form of DMP1 confirmed that DMP1-PG has a single GAG chain linked to $\mathrm{Ser}^{89}$ in mouse DMP1. Future studies involving transgenic mice carrying the S89G mutant transgene may provide valuable information regarding the biological functions of the GAG chain.

\section{Acknowledgment}

This work was supported by NIH grant DE005092 (C.Q.).

\section{References}

\footnotetext{
D’Souza, R.N., A. Cavender, G. Sunavala, J. Alvarez, T. Ohshima, A.B. Kulkarni, M. MacDougall (1997) Gene expression patterns of murine dentin matrix protein 1 (Dmp1) and dentin sialophosphoprotein (DSPP) suggest distinct developmental functions in vivo. J Bone Miner Res 12: 2040-2049.

-Feng, J.Q., L.M. Ward, S. Liu, Y. Lu, Y. Xie, B. Yuan, X. Yu, F. Rauch, S.I. Davis, S. Zhang, H. Rios, M.K. Drezner, L.D. Quarles, L.F.

Jeraman, S., K. Narayanan, J. Hao, C. Qin, A. George (2007) Matrix macromolecules in hard tissues control the nucleation and hierarchical assembly of hydroxyapatite. J Bio Chem 282: 1193-1204.

eorge, A., B. Sabsay, P.A. Simonian, A. Vei (1993) Characterization of a novel dentin matrix acidic phosphoprotein: implications for induction of biomineralization. J Bio Chem 268: 12624-12630.
} Bonewald, K.E. White (2006) Loss of DMP1 causes rickets and osteomalacia and identifies a role for osteocytes in mineral metabolism. Nat Genet 38: 1310-1315. 
He, G., S. Gajjeraman, D. Schultz, D. Cookson, C. Qin, W.T. Butler, J. Hao, A. George (2005) Spatially and temporally controlled biomineralization is facilitated by interaction between self-assembled dentin matrix protein 1 and calcium phosphate nuclei in solution. Biochemistry 44: 16140-16148.

Hirst, K.L., D. Simmons, J. Feng, H. Aplin, M.J. Dixon, M. MacDougall (1997) Elucidation of the sequence and the genomic organization of the human dentin matrix acidic phosphoprotein 1 (DMP1) gene: exclusion of the locus from a causative role in the pathogenesis of dentinogenesis imperfecta type II. Genomics 42: 38-45.

Lorenz-Depiereux, B., M. Bastepe, A. Benet-Pages, M. Amyere, J. Wagenstaller, U. MullerBarth, K. Badenhoop, S.M. Kaiser, R.S. Rittmaster, A.H. Shlossberg, J.L. Olivares, C. Loris, F.J. Ramos, F. Glorieux, M. Vikkula, H. Juppner, T.M. Strom (2006) DMP1 mutations in autosomal recessive hypophosphatemia implicate a bone matrix protein in the regulation of phosphate homeostasis. Nat Genet 38: 1248-1250.
MacDougall, M., T.T. Gu, X. Luan, D. Simmons, J. Chen (1998) Identification of a novel isoform of mouse dentin matrix protein 1: spatial expression in mineralized tissues. J Bone Miner Res 13: 422-431.

-Narayanan, K., R. Srinivas, A. Ramachandran, J. Hao, B. Quinn, A. George (2001) Differentiation of embryonic mesenchymal cells to odontoblast-like cells by overexpression of dentin matrix protein 1. Proc Natl Acad Sci USA 98: 4516-4521.

Ogbureke, K.U., L.W. Fisher (2007) SIBLING expression patterns in duct epithelia reflect the degree of metabolic activity. J Histochem Cytochem 55: 403-409.

Qin, C., J.C. Brunn, R.G. Cook, R.S. Orkiszewski, J.P. Malone, A. Veis, W.T. Butler (2003) Evidence for the proteolytic processing of dentin matrix protein 1: identification and characterization of processed fragments and cleavage sites. J Biol Chem 278: $34700-$ 34708.

Qin, C., O. Baba, W.T. Butler (2004) Post-translational modifications of sibling proteins and their roles in osteogenesis and dentinogenesis. Crit Rev Oral Biol Med 15: $126-$ 136.

Qin, C., B. Huang, J.N. Wygant, B.W. McIntyre, C.H. McDonald, R.G. Cook, W.T. Butler (2006) A chondroitin sulfate chain attached to the bone dentin matrix protein $1 \mathrm{NH}_{2}$-terminal fragment. J Biol Chem 281: 80348040 .
Tartaix, P.H., M. Doulaverakis, A. George, L.W. Fisher, W.T. Butler, C. Qin, E. Salih, M. Tan, Y. Fujimoto, L. Spevak, A.L. Boskey (2004) In vitro effects of dentin matrix protein-1 on hydroxyapatite formation provide insights into in vivo functions. J Biol Chem 279: 18115-18120.

Toyosawa, S., C. O’hUigin, H. Tichy, J. Klein (1999) Characterization of dentin matrix protein 1 gene in crocodilia. Gene 234: $307-$ 314.

Ye, L., M. MacDougall, S. Zhang, Y. Xie, J. Zhang, Z. Li, Y. Lu, Y. Mishina, J.Q. Feng (2004) Deletion of dentin matrix protein-1 leads to a partial failure of maturation of predentin into dentin, hypomineralization, and expanded cavities of pulp and root canal during postnatal tooth development. J Biol Chem 279: 19141-19148.

Ye, L., Y. Mishina, D. Chen, H. Huang, S.L. Dallas, M.R. Dallas, P. Sivakumar, T. Kunieda, T.W. Tsutsui, A. Boskey, L.F. Bonewald, J.Q. Feng (2005) Dmp1-deficient mice display severe defects in cartilage formation responsible for a chondrodysplasia-like phenotype. J Biol Chem 280: 6197-6203. 